\title{
ENRICHMENT ZONING STUDY FOR THE VERSATILE TEST REACTOR
}

\author{
Michael Jarrett and Florent Heidet \\ Argonne National Laboratory \\ 9700 S Cass Avenue, Lemont, IL 60439 \\ mjarrett@anl.gov, fheidet@anl.gov
}

\begin{abstract}
The primary mission of the Versatile Test Reactor (VTR) is to provide peak fast flux in excess of $4.0 \times 10^{15} \mathrm{n} / \mathrm{cm}^{2}$-s to support fuel and material testing. To achieve a high fast flux, it is beneficial to maximize the flux peaking in the center of the core. With a single enrichment zone, a highly peaked flux distribution produces a highly peaked power distribution. Coolant inlet orifices can be designed to handle the peaked power distribution but orifice design can be simplified if a more even radial power distribution can be achieved. An approach to reduce the power peaking factor is to use enrichment zoning, which would improve coolant flow homogeneity. Several alternative VTR core configurations are considered with two enrichment zones $(15 \mathrm{wt} \% \mathrm{Pu}$ and $20 \mathrm{wt} \% \mathrm{Pu})$. These alternative configurations require more assemblies to maintain reactivity than the reference VTR core, which leads to failure to achieve the design criterion for experimental fast flux with the target core power. Configurations using $20 \mathrm{wt} \%$ $\mathrm{Pu}$ with different fuel assembly designs having smaller and larger fuel volume fractions are also analyzed. The case having a larger fuel volume fraction reduces the number of fuel assemblies required for criticality, which keeps the experimental flux higher. Configurations with volume fraction zoning can slightly decrease the peaking factor while maintaining the desired fast flux, although some thermal hydraulic limits may not be satisfied. Volume fraction zoning configurations may offer benefits, but determining the feasibility of these configurations requires further thermal hydraulic design and analysis work beyond the scope of the present work.
\end{abstract}

KEYWORDS: sodium fast reactor, test reactor, enrichment zoning

\section{INTRODUCTION}

The Versatile Test Reactor (VTR) mission is to enable accelerated testing of advanced reactor fuels and materials required for advanced reactor technologies. As such, the goal of the design is to maximize the peak fast flux and the experimental volume exposed to a fast flux above $4.0 \times 10^{15} \mathrm{n} / \mathrm{cm}^{2}$-s. Existing Sodium-cooled Fast Reactor (SFR) technology is being leveraged to develop a $300 \mathrm{MWt}$ reactor that provides the desired experimental fast flux. $[1,2]$

The objective of maximizing the peak fast flux is most easily achieved by designing a reactor with a large flux gradient. With a single-enrichment core, this leads to a high radial power-peaking. It is possible to effectively cool a reactor with large power peaking ratios using different coolant flow restriction in the core. However, it is not possible to maintain a constant flow-to-power ratio in all assemblies when large 
differences in inlet orifice size exist. If the radial power profile and coolant flow are more evenly distributed, there could be less deviation from the desired coolant flow rates. Thus, there could be benefits in reducing the power peaking of the VTR while maintaining the desired experimental fast flux levels. Using multiple fuel enrichments (or fissile fractions) is one of the most effective ways to modify the radial power distribution.

\section{BACKGROUND}

The VTR fuel is a ternary metal alloy of uranium, plutonium, and zirconium. The reference fuel uses $5 \mathrm{wt} \%$ U-235 low-enriched uranium; the uranium enrichment is not modified in the present work. There is a constraint on the plutonium content dictated by prior fuel experience and on the uranium enrichment based on existing enrichment capabilities that limits the ultimate performance of the core. The plutonium content of the fuel should be no more than $20 \mathrm{wt} \%$ and the uranium enrichment is limited to about $5 \%$. These requirements are based on previous SFR operating experience with metal fuel, including the Fast Flux Test Facility (FFTF) and Experimental Breeder Reactor II (EBR-II). [3]

There are additional constraints placed on modification of the core. Relocation of the control rod and instrumented test assembly locations is not permitted, and the overall size of the reactor, including total number of assemblies, must not be changed. The only parameters that may be modified are the plutonium content of the fuel, the fuel volume fraction, or the number of fuel assemblies. Plutonium weight fraction zoning calculations are summarized in Section 3. Changes to assembly design, i.e. fuel volume fraction, are investigated in Section 4.

\section{ENRICHMENT ZONING WITH REACTOR-GRADE PLUTONIUM}

To flatten the radial power shape, there should be a gradient in the fuel enrichment, from low-fissile content near the center of the core to the highest fissile content at the periphery. Theoretically, a very flat power distribution could be achieved by using several enrichment zones, but this would introduce undesirable complications to the fuel fabrication requirements and fuel reloading patterns. For simplicity, configurations with two fissile levels are used for initial screening. If certain configurations are identified as potentially improving the core performance, these configurations can be refined by introducing a third fissile level.

The base VTR core configuration is shown in Fig. 1. Since the VTR core uses the maximum assumed plutonium content of $20 \mathrm{wt} \%$, the heterogeneous loading configurations must use a lower weight fraction zone in the center, rather than a higher weight fraction zone on the periphery. The loss in reactivity from using lower fissile content fuel in the center of the core must be offset by adding more driver assemblies. With a fixed reactor power of $300 \mathrm{MWt}$ distributed over more assemblies, the test fast fluxes are all reduced. Thus, it is inherently challenging to satisfy the experimental fast flux design criterion while simultaneously reducing power peaking with a lower weight fraction zone.

\subsection{Two-zone Configurations with 15 and 20 wt\% Plutonium Content}

Important core performance parameters for several two-zone configurations are compared to the base VTR core design in this section. These parameters are calculated using DIF3D-VARIANT [4], with $\mathrm{P}_{1}$ treatment of the flux, source, leakage, and scattering, $4^{\text {th }}$ and $6^{\text {th }}$ order spatial expansion of the flux and source, and 33 -group cross sections generated by $\mathrm{MC}^{2}-3$ [5]. These configurations are shown in Fig. 2. 


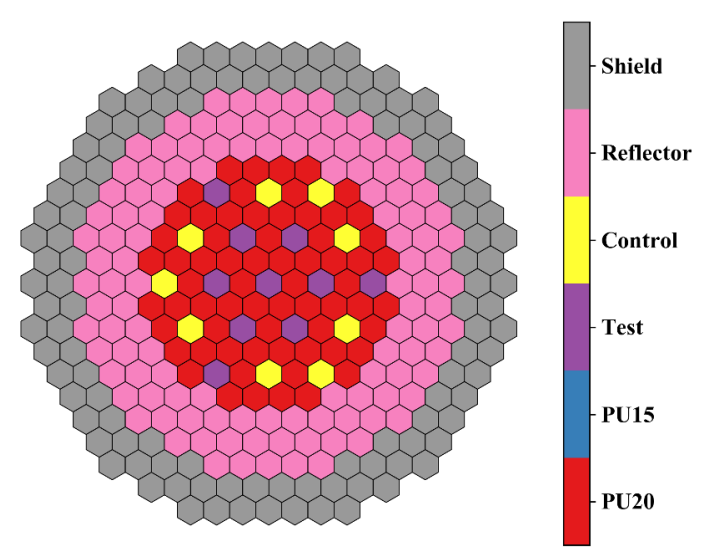

Figure 1. Base Configuration of VTR Core (20 wt\% Pu, 66 assemblies)
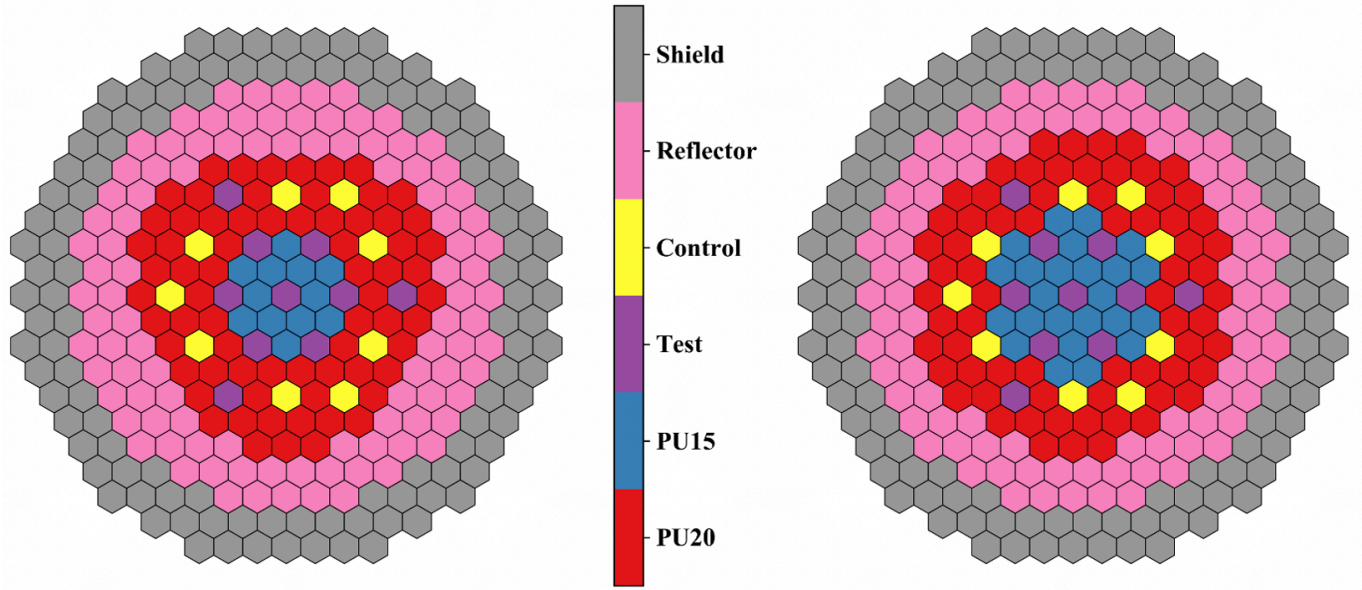

Shield

Reflector

Control

- Test

PU15

PU20
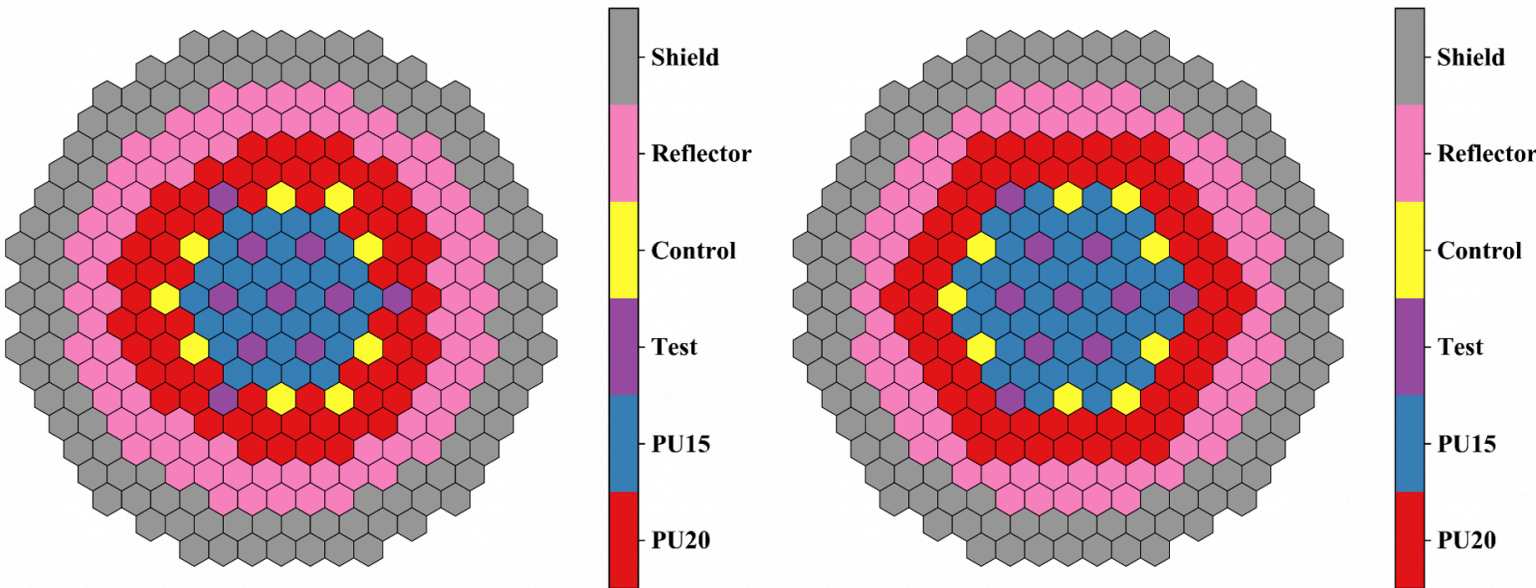

Figure 2. Two-zone 15/20 wt\% Pu configurations for the VTR core. Clockwise from top left: (i) Configuration A: 81 total assemblies, (ii) Configuration B: 92 total assemblies, (iii) Configuration D: 108 total assemblies, (iv) Configuration C: 96 total assemblies

The parameters of interest include power peaking factor, experimental fast flux, and average discharge burnup. For each configuration, a multicycle calculation is performed with automatic fuel reloading to maintain criticality [6]. Starting from a fresh core, the results from the first five cycles are discarded, and then parameters are averaged over the next 36 cycles. Beginning-of-cycle (BOC) values are used, but end- 
of-cycle (EOC) values are mostly similar with only a slightly higher experimental flux and slightly lower peaking factor.

Important core performance parameters for the various configurations shown in Fig. 2 are compared in Table 1. The two-zone configurations are designed to achieve a similar average discharge burnup to the reference. The reduction in maximum power from placing lower-enrichment fuel in the center of the core is somewhat offset by the reduction in average power caused by the additional fuel assemblies placed at the core periphery, which are necessary to compensate for the reduction in reactivity. As a result, the power peaking factor is reduced only marginally in the two-zone configurations. The radial power distributions for each configuration are shown in Fig. 3. In all two-zone configurations, the peak test fast flux (shown in Table 2) is far below the targeted $4.0 \times 10^{15} \mathrm{n} / \mathrm{cm}^{2}$-s.

Table 1. Two-zone configuration comparison: Average core performance parameters

\begin{tabular}{|c|c|c|c|c|c|}
\hline Parameter & $\begin{array}{c}\text { Single zone } \\
\text { (base case) }\end{array}$ & A & B & C & D \\
\hline Avg. 15 wt\% per cycle & 0.0 & 2.7 & 4.9 & 6.2 & 5.8 \\
\hline Avg. 20 wt\% per cycle & 15.5 & 12.8 & 10.9 & 10.6 & 7.0 \\
\hline Avg. discharge burnup (\%FIMA) & $4.80 \%$ & $4.90 \%$ & $4.80 \%$ & $4.50 \%$ & $5.90 \%$ \\
\hline Max power in 15 wt\% Pu (MW) & - & 4.31 & 3.92 & 3.76 & 3.51 \\
\hline Max power in 20 wt\% Pu (MW) & 6.26 & 4.74 & 4.14 & 3.75 & 3.14 \\
\hline Minimum power (MW) & 2.98 & 2.35 & 2.17 & 2.05 & 1.58 \\
\hline 15\%/20\% max power ratio & - & 0.91 & 0.95 & 1.00 & 1.12 \\
\hline $\begin{array}{c}\text { Average radial power peaking } \\
\text { (max-to-average ratio) }\end{array}$ & 1.47 & 1.36 & 1.34 & 1.27 & 1.20 \\
\hline $\begin{array}{c}\text { Average max-to-min } \\
\text { assembly power ratio }\end{array}$ & 2.20 & 2.04 & 1.94 & 1.82 & 2.60 \\
\hline Peak test fast flux (n/cm ${ }^{2}$-s) & $4.42 \mathrm{E}+15$ & $3.45 \mathrm{E}+15$ & $3.08 \mathrm{E}+15$ & $2.96 \mathrm{E}+15$ & $2.76 \mathrm{E}+15$ \\
\hline Number of fuel assemblies & 66 & 81 & 92 & 96 & 108 \\
\hline - with 15 wt\% Pu & 0 & 12 & 24 & 30 & 42 \\
\hline - with 20 wt\% Pu & 66 & 69 & 68 & 66 & 66 \\
\hline
\end{tabular}

In some configurations with a large number of fuel assemblies, there is not adequate space for a proper reflector region and shield region within the vessel. This is especially true for case $\mathrm{D}$. As a result, there may be an unacceptably high fluence on the vessel and activation of materials outside of the core in this configuration. This is another reason that this type of configuration is undesirable, in addition to the failure to meet experimental flux design criteria.

Table 2. Two-zone test fast flux: Average fast flux in experimental locations $\left(10^{15} \mathrm{n} / \mathrm{cm}^{2}-\mathrm{s}\right)$

\begin{tabular}{|c|c|c|c|c|c|}
\hline & $\begin{array}{c}\text { Single } \\
\text { zone }\end{array}$ & A & B & C & D \\
\hline Inner Test Location & 4.42 & 3.45 & 3.08 & 2.96 & 2.76 \\
\hline Middle Test Locations Avg. & 3.89 & 3.27 & 2.92 & 2.80 & 2.61 \\
\hline Outer Test Locations Avg. & 2.36 & 2.36 & 2.25 & 2.18 & 2.15 \\
\hline
\end{tabular}




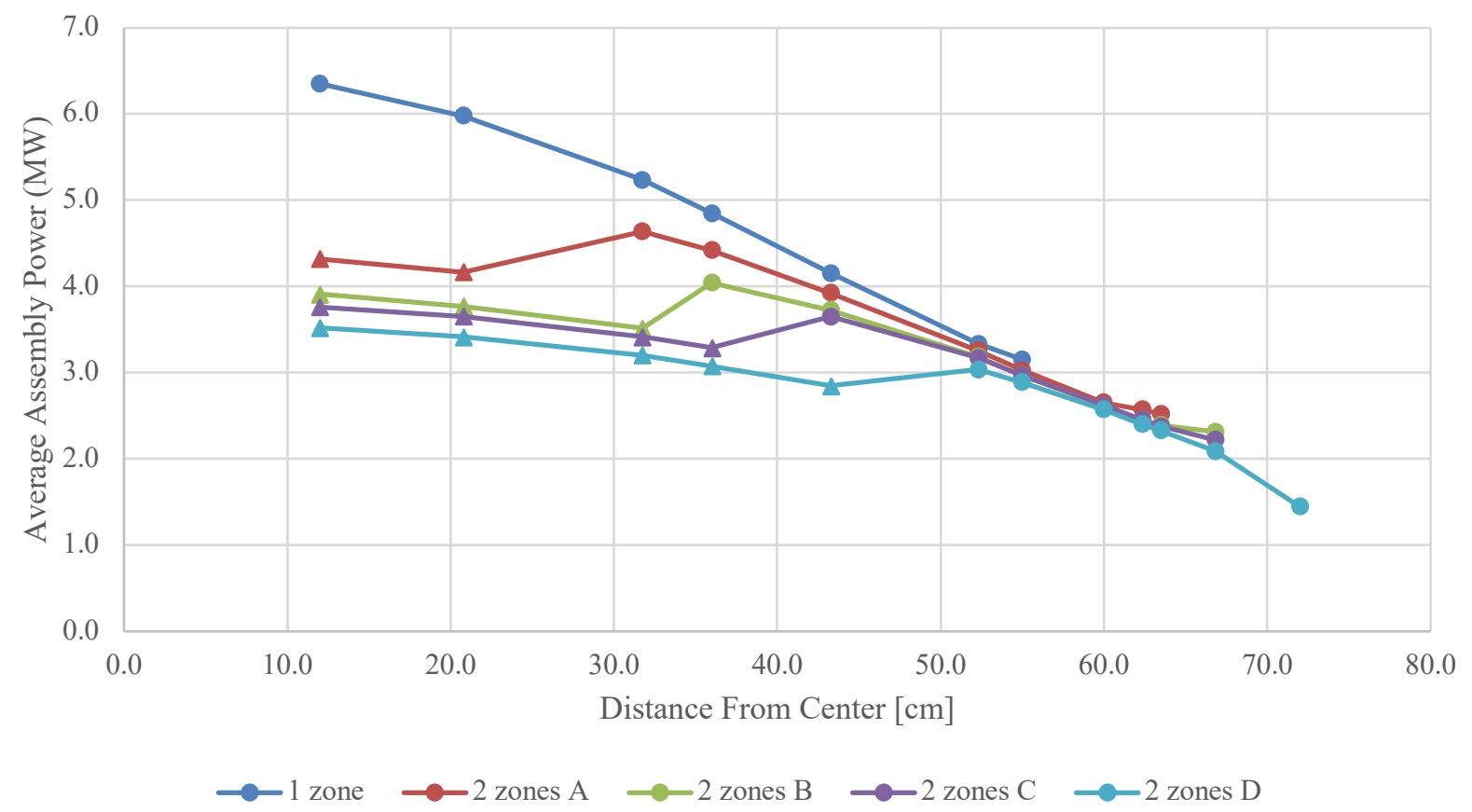

Figure 3. Comparison of radial power distribution for different configurations. Circle marker indicates $20 \mathrm{wt} \% \mathrm{Pu}$; triangle indicates $15 \mathrm{wt} \% \mathrm{Pu}$.

\section{ALTERNATE CONFIGURATIONS WITH VARYING FUEL VOLUME FRACTION}

Another way to adjust the radial power shape is to use multiple fuel assembly types, each with a different fuel volume fraction but the same fuel composition. The fuel volume fraction is the proportion of the assembly cross sectional area occupied by fuel. The overall power in an assembly is directly related to the flux, fissile fraction, and fuel volume fraction. Assemblies with lower fuel volume fraction will have higher coolant volume fraction, allowing more heat transfer and a higher maximum assembly power. The converse is true for assemblies with a higher fuel volume fraction. Technically, changing the fuel volume fraction may affect the spectrum enough that new broadgroup cross sections are required, but only a single set of cross sections are used for these calculations. This may introduce some quantitative error, but it is not significant enough affect the qualitative observations and trends highlighted.

Several configurations are evaluated in this section, each with some combination of four fuel types: $90 \%, 95 \%, 100 \%$, and $110 \%$ of the reference fuel volume fraction. The fuel volume fractions are $0.339,0.358,0.377$, and 0.414 , respectively. The configurations considered are shown in Fig. 4.

The core performance parameters are compared to the base VTR core configuration in Table 3. The radial power distribution is shown in Fig. 5. The power peaking is reduced marginally, from 1.47 to $(1.30,1.37)$. The test fast fluxes, given in Table 4 , are sufficient in all cases. For configuration $X$ the average maximum assembly power in the $110 \%$ volume fraction zone is 5.5 MW, which may be beyond the limit for that assembly type. A more detailed thermal hydraulic analysis would be required to make a judgment for configuration $\mathrm{X}$. Configuration $\mathrm{X}$ and $\mathrm{Z}$ require more than 15 fresh assemblies per cycle, while configuration $\mathrm{Y}$ only requires 12.3. 

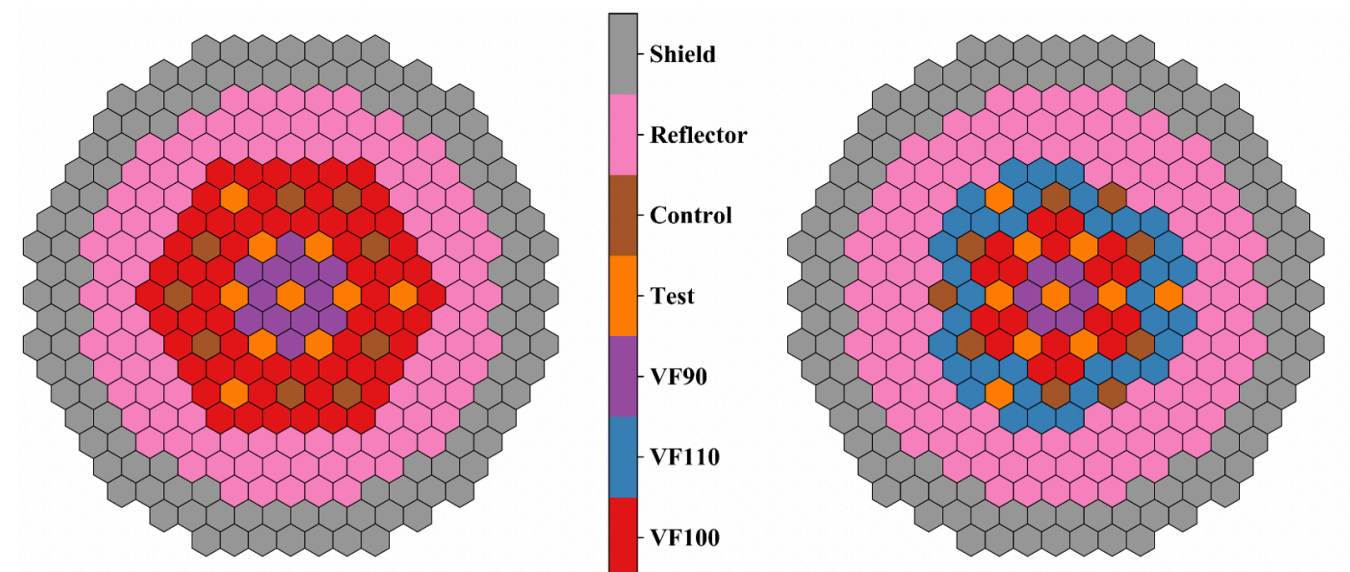

- Shield

Reflector

Control

- Test

-VF95

-VF110

VF100
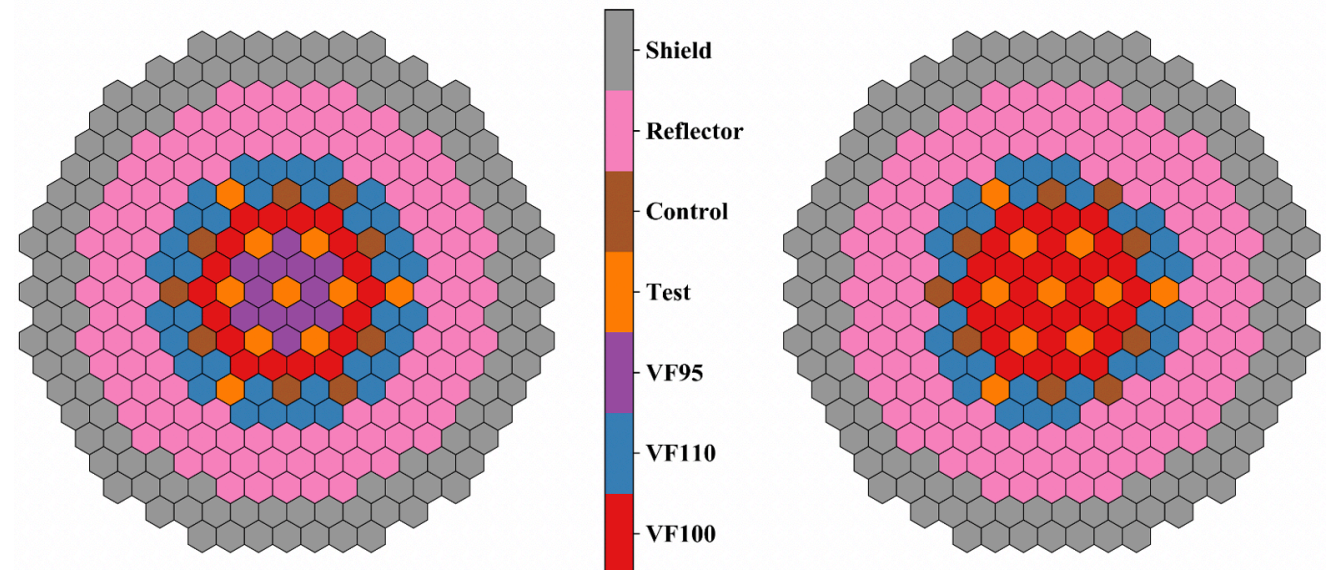

Shield

Reflector

Control

Test

-VF90

VF110

- VF100

Figure 4. Fuel type zones for varying fuel volume fractions. Clockwise from top left:

(i) Configuration W, 72 assemblies, (ii) Configuration X, 60 assemblies, (iii) Configuration Z, 60 assemblies, (iv) Configuration Y, 66 assemblies

Table 4. Volume fraction (VF) zoning comparison: Average core performance parameters

\begin{tabular}{|c|c|c|c|c|c|}
\hline & Single zone & W & X & Y & Z \\
\hline Avg. 20 wt\% per cycle & 15.5 & 11.7 & 5.9 & 3.9 & 9.6 \\
\hline Avg. high VF per cycle & 0.0 & 0.0 & 7.3 & 5.4 & 6.1 \\
\hline Avg. low VF per cycle & 0.0 & 3.9 & 2.0 & 3.0 & 0.0 \\
\hline Avg. discharge burnup (\% FIMA) & $4.80 \%$ & $4.96 \%$ & $4.73 \%$ & $5.98 \%$ & $4.57 \%$ \\
\hline maximum power (MW) & 6261.3 & 5166.7 & 6175.7 & 5778.1 & 6540.8 \\
\hline minimum power (MW) & 2984.5 & 2530.4 & 3290.6 & 3058.6 & 3358.7 \\
\hline Avg. radial power peaking & 1.47 & 1.36 & 1.30 & 1.33 & 1.37 \\
\hline Avg. max-to-min power ratio & 2.20 & 2.12 & 1.83 & 1.85 & 1.94 \\
\hline Peak test fast flux (n/cm ${ }^{2}$-s) & $4.42 \mathrm{E}+15$ & $4.01 \mathrm{E}+15$ & $4.45 \mathrm{E}+15$ & $4.16 \mathrm{E}+15$ & $4.53 \mathrm{E}+15$ \\
\hline Number of fuel assemblies & 66 & 72 & 60 & 66 & 60 \\
\hline - With 110\% Fuel VF & 0 & 0 & 36 & 36 & 30 \\
\hline - With 100\% Fuel VF & 66 & 60 & 18 & 18 & 30 \\
\hline - With 95\% Fuel VF & 0 & 0 & 6 & 12 & 0 \\
\hline - With 90\% Fuel VF & 0 & 12 & 0 & 0 & 0 \\
\hline
\end{tabular}




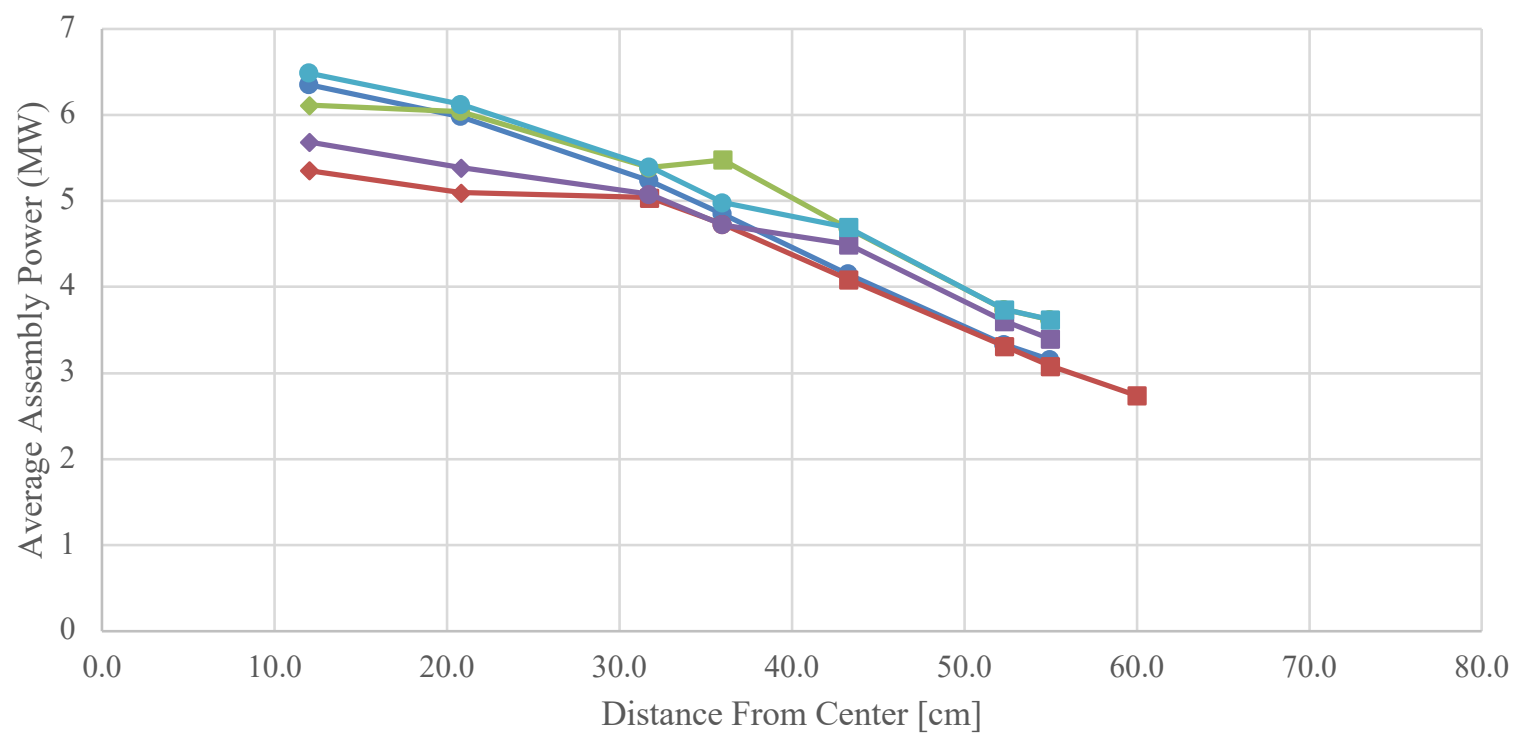

$\longrightarrow 1$ zone $\longrightarrow 2$ zones $\mathrm{W} \longrightarrow 3$ zones $\mathrm{X} \quad-3$ zones $\mathrm{Y} \quad-2$ zones $\mathrm{Z}$

Figure 5. Radial power distribution for volume fraction zoning. Circle marker indicates reference VF; square marker indicates high VF; diamond marker indicates low VF.

Table 3. Six-zone test fast flux: Average fast flux in experimental locations $\left(10^{15} \mathrm{n} / \mathrm{cm}^{2}-\mathrm{s}\right)$

\begin{tabular}{|c|c|c|c|c|c|}
\hline & $\begin{array}{c}\text { Single } \\
\text { zone }\end{array}$ & 2 zones W & 2 zones X & 2 zones Y & 2 zones Z \\
\hline Inner Test Location & 4.42 & 4.01 & 4.45 & 4.16 & 4.53 \\
\hline Middle Test Locations Avg. & 3.89 & 3.65 & 3.98 & 3.75 & 3.99 \\
\hline Outer Test Locations Avg. & 2.36 & 2.42 & 2.50 & 2.38 & 2.49 \\
\hline
\end{tabular}

\section{CONCLUSIONS}

The goal of this work is to explore whether the performance of the VTR core could be improved by using multiple enrichment zones or multiple fuel types. The VTR fuel design uses a single fuel composition with ternary U-20Pu-10Zr fuel. The uranium enrichment is $5 \mathrm{wt} \% \mathrm{U}-235$. The plutonium content of the fuel is capped at $20 \mathrm{wt} \%$ because there is not sufficient prior operating experience with metal fuel under relevant conditions to support ternary alloys with higher plutonium content. This limit constrains the possible range of alternate configurations, thereby limiting the effectiveness of using several fissile levels. Only heterogeneous configurations with average plutonium content lower than $20 \mathrm{wt} \%$ are possible; these require adding fuel assemblies to maintain reactivity, which flattens flux shape along with the power shape. As a result, it is not possible within the constraints of this study to simultaneously achieve both (1) the reduced power peaking sought in enrichment zoning and (2) the design criterion of $4.0 \times 10^{15} \mathrm{n} / \mathrm{cm}^{2}$-s fast flux in a test assembly.

Flattening of the power shape through variation of the assembly fuel volume fraction was also attempted. This approach was more effective than the fissile content zoning approach. All four configurations achieved mildly reduced power peaking while maintaining the required test fast flux. In cases $\mathrm{X}$ and $\mathrm{Z}$, there may be unacceptably thin margins between the assembly powers and their designed limits. Case Y appears to meet 
all of the criteria, with a high average discharge burnup and sufficient test fast flux, and the radial peaking factor is reduced by $32 \%$. However, there is a slight reduction of the maximum fast flux observed.

Overall, flattening of the flux shape is somewhat undesirable because to maintain similar peak performance (in the central test location), the overall core power would need to be increased above the desired $300 \mathrm{MWth}$. None of the configurations with multiple enrichment zones achieve the design criterion for test fast flux. All of the multiple assembly type cases achieve the desired fast flux, but the peaking factor is not reduced enough to make manufacturing several fuel compositions worthwhile.

Fissile content zoning would be more effective if one or both of the two fuel composition constraints (on uranium enrichment and plutonium weight content) were relaxed. If higher enrichments could be used, the power shape could be flattened and the number of fuel assemblies could be slightly reduced or kept the same. At a constant total core power, this would allow a lower peaking factor while maintaining a high experimental fast flux in the central test location.

If the total core power could be increased marginally above $300 \mathrm{MW}$, then the fast flux could effectively be scaled up by a similar ratio to the power scaling. This would make it easier to meet the fast flux criterion with a lower fissile content zone in the center of the core.

\section{ACKNOWLEDGMENTS}

The submitted manuscript has been created by UChicago Argonne, LLC, Operator of Argonne National Laboratory ("Argonne"). Argonne National Laboratory's work was supported by the U.S. Department of Energy, Office of Nuclear Energy under contract DE-AC02-06CH11357.

The work reported in this summary is the results of R\&D studies supporting a VTR concept, cost, and schedule estimate for DOE-NE to make a decision on procurement in the future. As such, it is predecisional.

\section{REFERENCES}

1. F. Heidet, G. Youinou, J. Roglans-Ribas, "Overview of the Versatile Test Reactor Core Design Activities" Transactions of the 2019 ANS Annual Meeting, Minneapolis, MN, USA. 10-13 June 2019.

2. F. Heidet, "Current Status of the VTR Core Design," Proceedings of the RRFM/IGORR 2019 conference, 24-28 March 2019, Jordan.

3. D.C. Crawford and D.L. Porter and S.L. Hayes, "Fuels for sodium-cooled fast reactors: US perspective," Journal of Nuclear Materials, 371, 202-231 (2007).

4. G. Palmiotti et al, "Variational nodal transport methods with anisotropic scattering," Nuclear Science and Engineering, 115, 233-243 (1993).

5. C. Lee, W. S. Yang, "MC2-3: Multigroup Cross Section Generation Code for Fast Reactor Analysis," ANL/NE-11-41 Rev.3, August 31 (2018).

6. M. Jarrett and F. Heidet, "Automated Fuel Management Optimization for Fast Reactors," Proceedings of PHYSOR 2020, Cambridge, UK (2020). 